\title{
Perspectivas da geração e aplicação da energia solar fotovoltaica no Brasil: uma revisão da literatura (2015-2019)
}

O presente estudo tem por objetivo realizar uma revisão na literatura no período de 2015 a 2019 sobre a geração e uso da energia solar fotovoltaica no Brasil. Foram realizadas pesquisas por artigos científicos em bases de dados como SCOPUS e SCIELO, a partir dos critérios de inclusão: publicações nacionais e internacionais na forma de artigos científicos originais e artigos publicados em periódicos avaliados por pares. E posteriormente utilizados palavras chaves: energia fotovoltaica (photovoltaic energy) e sistemas fotovoltaicos (photovoltaic systems). Também foram consideradas as publicações oficiais sobre o balanço energético nacional (Empresa de Pesquisa Energética - EPE). A conclusão é que a tecnologia fotovoltaica vem sendo mais utilizada em sistemas híbridos de geração de energia em sistemas conectados à rede convencional de energia e principalmente, em combinação com a energia eólica. Apesar de ser uma fonte energética alternativa com uso bastante disseminado, ainda enfrenta entrave devido, principalmente, aos elevados custos para financiamento dos painéis solares.

Palavras-chave: Energia Solar Fotovoltaica; Sistemas Fotovoltaicos; Energia Alternativa.

\section{Perspectives of the generation and application of photovoltaic solar energy in Brazil: a literature review (2015-2019)}

The present study has by objective performs a review at literature in the period from 2015 to 2019 on the generation and use gives photovoltaic solar energy at the Brazil. Were realized researches by scientific articles in data base as SCOPUS and SCIELO, the starting of the criterions of inclusion: publications national and international in the form of scientific articles original and articles published in periodic peer reviewed. And posteriorly used keywords: photovoltaic energy and photovoltaic systems. It was also considered the publications official over the national energy balance (Energy Research Company - EPE). The conclusion is that the photovoltaic technology come being more used in hybrid systems of electricity generation in systems connected the network conventional of energy with use quite disseminated, still encounter obstacle due, principally, to the elevated costs for financing of the solar panels.

Keywords: Photovoltaic Solar Energy; Photovoltaic Systems; Alternative Energy

Topic: Sistemas de Energia Sustentável

Reviewed anonymously in the process of blind peer.
Received: 17/04/2021

Approved: 18/05/2021
Esdras Alex Freire de Oliveira

Universidade Regional do Cariri, Brasi

http://lattes.cnpq.br/4693470747465264

esdras-alex@hotmail.com

José Gonçalves de Araújo Filho

Universidade Regional do Cariri, Brasil

http://lattes.cnpq.br/4909589002760742

araujo.filho@urca.br
Referencing this:

OLIVEIRA, E. A. F.; ARAÚJO FILHO, J. G.. Perspectivas da geração e aplicação da energia solar fotovoltaica no Brasil: uma revisão da literatura (2015-2019). Revista Ibero Americana de Ciências Ambientais, v.12, n.5, p.435-450, 2021. DOI: http://doi.org/10.6008/CBPC2179-6858.2021.005.0035 


\section{INTRODUÇÃO}

A avaliação de recursos alternativos para atender à crescente demanda de eletricidade é um aspecto essencial no planejamento do desenvolvimento dos países em desenvolvimento (VIANA et al., 2018). Entre os diversos recursos disponíveis no mercado para produção de eletricidade, podemos considerar as fontes de energia renováveis como alternativa ambientalmente sustentável. Uma destas fontes de energia sustentáveis é a energia solar fotovoltaica, que é obtida pela conversão da luz solar em eletricidade por meio de uma tecnologia baseada no efeito fotoelétrico.

O mercado solar mundial é dominado por painéis fotovoltaicas produzidas a partir de matériaprima a base de silício cristalino. A participação da energia solar na matriz energética brasileira ainda é pequena, mas o país está, aos poucos, tomando conta da sua relevância. Segundo Silva et al. (2019) a produção de eletricidade utilizando sistemas fotovoltaicos apresenta benefícios ambientais bastantes significativos e em termos de segurança energética, sendo que os dispositivos utilizados para conversão de radiação solar em energia elétrica são de fácil aplicação, não produzem ruídos e tampouco elementos poluentes durante sua operação. De acordo com Souza et al. (2019), o Brasil apresenta condições naturais favoráveis para a geração de energia elétrica a partir de fonte solar, visto que o país possui níveis de irradiação bem maiores que outros países, onde os projetos para aproveitamento desse tipo de energia são largamente disseminados, como Alemanha, França e Espanha. A incidência de irradiação solar média no Brasil é na faixa de $1500-2500 \mathrm{kWh} / \mathrm{m}^{2}$, sendo bem maiores que as irradiações apresentadas por esses países europeus: Alemanha $\left(900-1250 \mathrm{kWh} / \mathrm{m}^{2}\right)$, França $\left(900-1650 \mathrm{kWh} / \mathrm{m}^{2}\right)$ e Espanha $(1200-1850$ $\mathrm{kWh} / \mathrm{m}^{2}$ ) (AQUINO et al., 2019).

Somente a partir da publicação da Resolução Normativa n.o 482, em 2012, pela Agência Nacional de Energia Elétrica (ANEEL), que iniciaram os primeiros incentivos no Brasil para a geração descentralizada e distribuída da energia solar fotovoltaica, o que certamente possibilitou uma maior inserção dessa tecnologia no mercado nacional. Nessa perspectiva, o presente estudo tem por objetivo realizar uma revisão na literatura no período de 2015 a 2019 sobre a geração e uso da energia solar fotovoltaica no Brasil.

A procura por fontes de energia alternativas, menos poluentes e menos agressivas ao ambiente, para suprir as necessidades energéticas do atual estágio do desenvolvimento humano, teve como motivação a necessidade de diminuir a emissão dos gases de efeito estufa (GEE); e o avanço de pesquisas relacionadas ao campo de energias renováveis (TORRES, 2012, p.27), como também pelo esgotamento das reservas de combustíveis fósseis, pela baixa eficiência global e pelo os impactos ambientais ocasionados pelas usinas energéticas convencionais (PIRES et al., 2019). É neste cenário onde o desenvolvimento sustentável está sendo considerada como uma questão importante no processo de restruturação da matriz energética mundial, que a energia solar fotovoltaica se configura como um caminho alternativo e promissor. Para Aquino et al. (2019) esse tipo de fonte de energia pode oferecer inúmeras vantagens na geração, transmissão e distribuição de energia elétrica, trazendo de forma perceptível, confiabilidade técnica, melhor custo-benefício, baixo impacto ambiental, que naturalmente chega o mais próximo possível 
de um denominador comum entre os termos: custo da energia elétrica, demanda energética crescente e desenvolvimento sustentável.

Os sistemas fotovoltaicos são capazes de gerar energia elétrica a partir da utilização de células fotovoltaicas, tais células são constituídas de materiais que transformam radiação solar diretamente em energia elétrica, processo denominado de efeito fotovoltaico (AQUINO et al., 2019). O efeito fotovoltaico consiste em um fenômeno físico que possibilita a conversão direta da luz solar em eletricidade, sendo que tal fenômeno ocorre quando a luz ou a radiação eletromagnética do Sol, incide sobre uma célula composta de materiais semicondutores com propriedades específicas (VILLALVA et al., 2012).

Historicamente a descoberta dessa fonte de energia data desde o início do século XIX, quando o cientista e físico Edmond Becquerel (1820-1891), pai de Henri Becquerel (1852-1908), conhecido pelos estudos em radioatividade, observou pela primeira vez o efeito fotovoltaico, em 1839, através de uma solução química envolvendo selênio (VILLALVA et al., 2012). Mais tarde, em 1877, esse mesmo experimento foi estudado por outros cientistas que constataram o mesmo resultado utilizando selênio no estado sólido, onde surgiram as primeiras células solares a base de selênio, com eficiência energética inferior a 1\%. No entanto, o primeiro dispositivo viável foi desenvolvido em 1953, nos Laboratórios Bell, nos EUA, em um substrato de silício (SAUER et al., 2006), desde então a produção de células solares fotovoltaicas passaram a ser fabricadas a base de silício, por ser um material condutor eficiente com grande abundância na natureza e bem mais barato que outros tipos de materiais.

O silício ( $\mathrm{Si}$ ) é um metal semicondutor, extraído do mineral quartzo $\left(\mathrm{SiO}_{2}\right)$ (sua principal fonte), que no estado puro possui cor cinza e baixa condutividade elétrica. Este elemento químico é bastante utilizado na produção de células solares na forma de silício grau solar (SiGS), sendo que esse material é obtido a partir de processos de purificações e dopagem do silício grau metalúrgicos (SiGM). O SiGM, matéria-prima de partida e a mais usada na produção do SiGS, possui um teor de Si entre 98,70 e 99,50\%, sendo obtido através da redução carbotérmica do quartzo por agentes redutores em forno mecânico a arco elétrico (CETEM, 2019).

Conforme Machado e Miranda (2015) as principais células solares encontradas atualmente no mercado são: as de silício cristalino (c-Si), no qual se subdividem em dois tipos de materiais denominados: silício monocristalino e policristalino; e as de silício amorfo (a-Si). O silício monocristalino é obtido através do processo de Czochralski (CZ), que é um método de crescimento de monocristais de semicondutores em um reator, onde são adicionados em proporções especificas os elementos dopantes, geralmente fósforo e boro, para potencializar as propriedades condutoras do material (CETEM, 2019).

Segundo Torres et al. (2019) o silício policristalino é obtido a partir da fusão de silício em moldes, sendo que o processo de fabricação é bem mais barato e econômico do que aquele empregado no silício monocristalino, por não depender de tanta complexidade na produção dos cristais. Já o sílico amorfo é considerado uma forma alotrópica de silício, sem orientação cristalográfica, produzido pelo processo PECVD (plasma-enhanced chemical vapor deposition), onde o material é depositado em uma câmera a vácuo contento plasma (substrato formado através da eletrização de uma mistura gasosa de silano e 
hidrogênio) (CETEM, 2019).

Segundo Villalva e Gazoli (2012) as células fotovoltaicas de silício monocristalino são consideradas as células solares com maior eficiência presente no mercado atualmente, podendo alcançar níveis em torno de 15 a 18\% de aproveitamento da radiação solar, porém, tem um custo de produção mais elevado que outros tipos de células. Ainda conforme esses autores, outras células fotovoltaicas como a de silício policristalino e amorfo apresentam custos de fabricação e eficiência energética inferiores a célula de silício monocristalino, sendo que os níveis de eficiências giram em torno de 13 a 15\% para as células de silício policristalino e 5 a $8 \%$ para células de silício amorfo.

A produção de energia elétrica a partir de um sistema fotovoltaico pode ser utilizada de forma isolada (também chamado de sistemas fotovoltaicos autônomos) ou conectada à rede pública de energia (denominado sistemas fotovoltaicos conectados à rede elétrica). No primeiro caso, a energia elétrica produzida é armazenada em baterias para posteriormente ser utilizada. No segundo caso, a energia elétrica gerada é lançada diretamente na rede pública de energia, podendo ser utilizada tanto para reduzir o consumo de energia elétrica ou mesmo para gerar excedente de energia. O sistema fotovoltaico autônomo é empregado em locais não atendidos anteriormente por uma rede elétrica, como: residências em zona rurais, praias, ilhas, na sinalização de estradas, na alimentação de sistemas de telecomunicações, no carregamento de baterias de veículos elétricos, dentre outras aplicações. Já o sistema fotovoltaico conectado à rede elétrica funciona em paralelismo com o sistema público de eletricidade, que diferentemente do sistema autônomo, essa forma de produção de energia é empregada em locais já atendidos por energia elétrica como, por exemplo, as residências familiares existente em uma zona urbana (VILLALVA et al., 2012).

De acordo com Didoné et al. (2014), o interesse na aplicação da energia solar fotovoltaica no Brasil começou a ser discutida no início da década passada, porém, o primeiro sistema fotovoltaico foi implantado no país em 1997 pelo Grupo de Pesquisa Estratégica em Energia Solar (FV-UFSC) na Universidade Federal de Santa Catarina (UFSC). No Brasil, até o início de 2012, a principal barreira que dificultava a geração de energia solar fotovoltaica de forma distribuída e descentralizada era a inexistência de regulamentação e de normas técnicas, o que dificultava o advento de indústrias e de mercados voltados para os sistemas de geração de energia em baixa tensão, que certamente são um importante nicho de aplicação da energia solar fotovoltaica (VILLALVA et al., 2012). Esse estudo caracteriza-se como uma pesquisa de natureza exploratória e descritiva, realizada através de revisão bibliográfica durante o período histórico entre 2015 a 2019, sobre o contexto da energia solar fotovoltaica no Brasil.

Segundo Gil (2017) o propósito da pesquisa exploratória é proporcionar uma maior familiaridade com o objeto de estudo, com vista a torna-los mais explícitos, sendo que o planejamento desse tipo de pesquisa tende a ser flexível em razão de considerar os mais diversos aspectos do fenômeno investigado. Ainda segundo esse autor as pesquisas descritivas visa descrever as características peculiares de determinado fenômeno, população ou mesmo o estabelecimento de relações entre variáveis. A pesquisa bibliográfica, conforme Azevedo (2016), tem como objetivo conceder uma visão abrangente das fontes 
consultadas sobre um determinado tema de estudo e tem características de investigação científica.

Para elaboração dos resultados desse estudo foram consultados artigos científicos publicados em periódicos e publicações oficiais sobre o balanço energético nacional (Empresa de Pesquisa Energética EPE). Os artigos consultados foram coletados em duas bases de dados: Scopus, Scientific Electronic Library Online (SCIELO), utilizando os seguintes critérios de inclusão: publicações nacionais e internacionais na forma de artigos científicos originais; artigos publicados em periódicos avaliados por pares; publicações cientificas enquadradas entre o período de janeiro/2012 a dezembro/2019. Para consulta dos artigos, realizou-se a busca nas bases de dados com as seguintes palavras-chave: energia fotovoltaica (photovoltaic energy), sistemas fotovoltaicos (photovoltaic systems), direcionada para pesquisas desenvolvidas e aplicadas no território nacional.

\section{RESULTADOS E DISCUSSÃO}

As consultas realizadas nas plataformas digitais citadas nesse estudo, totalizaram-se em cerca de 174 manuscritos encontrados. Na base de dados SCIELO foram encontrados 101 artigos científicos e na base de dados SCOPUS resultou em 73 artigos. Logo após a realização do processo de avaliação dos artigos científicos para verificar a adequação dos conteúdos das publicações ao contexto da proposta de pesquisa, obteve-se um total de 35 artigos selecionados, conforme pode ser verificado no quadro a seguir.

Quadro 1: Resultado da seleção dos artigos científicos

\begin{tabular}{|l|l|l|}
\hline BASE DE DADOS & TOTAL DE REFERÊNCIAS & REFERÊNCIAS SELECIONADAS \\
\hline SCIELO & 101 & 20 \\
\hline SCOPUS & 73 & 15 \\
\hline TOTAL & $\mathbf{1 7 4}$ & $\mathbf{3 5}$ \\
\hline
\end{tabular}

Os resultados dos artigos pesquisados tiveram uma maior predominância dos achados no periódico Brazilian Archives of Biology and Technology com $40 \%$ de um total de 35 manuscritos. Em relação aos períodos analizados, esse periódico apresentou 40\% dos achados em 2018 e 46\% em 2019, sendo indexado as principais bases de dados como Scopus (RELX Group conhecida como Reed Elsevier) e Scientific Electronic Library Online (SciELO). Outro periódico com maior representatividade nos achados foi Renewable Energy com $11 \%$ dos artigos, tendo como principais indexações: Web of Science (da empresa Clarivates), Scopus (Reed Elsevier). Os demais periódicos, não menos importante em questão de reputação no meio acadêmico, tiveram menos de $6 \%$ dos articos selecionados para análise.

Considerando as características do elenco de artigos encontrados, é importante frisar que 91\% dos achados estavam publicados no idioma inglês, sendo que parte dessas publicações utilizam como metodologias a simulação matemática aplicados a sistemas híbridos de energia. Tais estudos visavam a complementação energética em sistemas convencionais de energia utilizando a tecnologia fotovoltaica, bem como a combinação dessa tecnologia a outras fontes de energia renovavél, principalmente, a aplicação com a energia eólica. Outra caracterítica inerente aos achados, é que $31 \%$ dos manuscritos foram aplicados no estado do Paraná, especificamente na cidade de Curitiba, com citação a Universidade Federal de Tecnologia do Paraná (UTFPR). 
Desde 2015, a micro e mini geração de energia solar fotovoltaica vem ganhando destaque na matriz energética brasileira, apresentando taxa de crescimento significativa em razao de incentivos por ações regulatórias. Em relação ao período 2019-2015, a taxa de geração e potência instalada obteve, respectivamente, um acréscimo de 98,79\% (1639 GWh) e 99,33\% (1978,7 MW), porém, atualmente a participação da fonte de energia solar na matriz enegética do país ainda apresenta percentual inferior a $2 \%$ da capacidade instalada (BEM, 2020). Analisando os cenários para geração de eletricidade a parti de energia solar fotovoltaica, Urbanetz et al. (2019), verificou que até 2025 (seguindo a tendência mundial de crescimento de 30\%), o Brasil teria potência de energia fotovoltaica instalada de aproximadamente 75,6 GWp, apresentando taxa de geração de 98,3 TWh de energia elétrica e demanda estimada para o referido período de 800 TWh, o que resultaria na contribuição para matriz elétrica brasileira de 12,3\%.

Conforme Carstens et al. (2019) apesar da baixa representatividade da energia fotovoltaica no sistema energético brasileiro, essa tecnologia ainda apresenta cenários de crescimento favoráveis, sendo que os leilões realizados em 2015 "firmaram um total de 33 projetos de geração solar fotovoltaica, com capacidade máxima de 1115 MW e deverá entrar em operação a partir de novembro de 2018, com prazo contratual de 20 anos de fornecimento de energia". Os leilões de energia são de grande importância para supri à demanda energética brasileira em razão da produção em larga escala e na prevenção da escassez de energia no que tange a redução das incertezas (pois tratar-se de projetos de longo prazo), além disse os leilões proporcionam um melhor controle de custos, devido ao aproveitamento de ofertas com menores preços de mercado.

Nobre et al. (2019) realizou um estudo sobre redes inteligentes de geração, distribuição e consumo de energia elétrica como alternatica para inclusão econômica e adaptação as mudanças climaticas no Nordeste brasileiro, através de sistemas fotovoltaicos. Neste ensaio, os autores verificaram que implementação de micro-usinas fotovoltaicas distribuídas em áreas degradadas constitue uma excelente alternativa para desenvolvimento socioeconomico dessa região e por se tratar de áreas improprias para a desevolver atividades agrícolas, estar livre de conflitos de interesse. O aproveitamento dessas áreas possibilitaria vatagens tantos na geração de emprego e renda para população local como proporcionaria a produção de eletricidade através dos paineis solares instalados. No quadro a seguir são apresentados os artigos selecionados para essa revisão com indicação dos autores, ano de publição e títulos das publicações.

Quadro 2: Artigos selecionados

\begin{tabular}{|l|l|l|l|}
\hline Autores & Ano & Periódico & Títulos \\
\hline Urbanetz et al. & 2019 & $\begin{array}{l}\text { Brazilian Archives of Biology } \\
\text { and Technology }\end{array}$ & $\begin{array}{l}\text { Current panorama and 2025 scenario of photovoltaic solar energy in } \\
\text { Brazil }\end{array}$ \\
\hline Carstens et al. & 2019 & Energy Policy & $\begin{array}{l}\text { Challenges and opportunities for the growth of solar photovoltaic } \\
\text { energy in Brazil }\end{array}$ \\
\hline Nobre et al. & 2019 & $\begin{array}{l}\text { International Journal of } \\
\text { Climate Change Strategies and } \\
\text { Management }\end{array}$ & $\begin{array}{l}\text { Solar smart grid as a path to economic inclusion and adaptation to } \\
\text { climate change in the Brazilian Semiarid Northeast }\end{array}$ \\
\hline Souza et al. & 2016 & $\begin{array}{l}\text { Energy Research and Social } \\
\text { Science }\end{array}$ & $\begin{array}{l}\text { Towards a sociology of energy and globalization: Interconnectedness, } \\
\text { capital, and knowledge in the Brazilian solar photovoltaic industry }\end{array}$ \\
\hline Silva et al. & 2019 & $\begin{array}{l}\text { Impact Assessment and } \\
\text { Project Appraisal }\end{array}$ & $\begin{array}{l}\text { Environmental licensing and energy policy regulating utility-scale solar } \\
\text { photovoltaic installations in Brazil: status and future perspectives }\end{array}$ \\
\hline
\end{tabular}




\begin{tabular}{|c|c|c|c|}
\hline Rosa et al. & 2017 & Sustainability & $\begin{array}{l}\text { Complementarity of hydro, photovoltaic, and wind power in Rio de } \\
\text { Janeiro State }\end{array}$ \\
\hline $\begin{array}{l}\text { Figueirêdo Neto } \\
\text { et al. }\end{array}$ & 2019 & Energy Policy & $\begin{array}{l}\text { Photovoltaic energy in the enhancement of indigenous education in } \\
\text { the Brazilian Amazon }\end{array}$ \\
\hline Jong et al. & 2019 & Renewable Energy & $\begin{array}{l}\text { Estimating the impact of climate change on wind and solar energy in } \\
\text { Brazil using a South American regional climate model }\end{array}$ \\
\hline Schmidt et al. & 2016 & Renewable Energy & $\begin{array}{l}\text { An optimal mix of solar PV, wind and hydro power for a low-carbon } \\
\text { electricity supply in Brazil }\end{array}$ \\
\hline Carvalho et al. & 2018 & $\begin{array}{l}\text { Engenharia Sanitaria e } \\
\text { Ambiental }\end{array}$ & $\begin{array}{l}\text { Sizing optimization in solar photovoltaic water pumping system: } \\
\text { Model validation in a pilot unit located in the rural community of Rio } \\
\text { Belo, Orleans/SC, Brazil }\end{array}$ \\
\hline Pereira et al. & 2018 & $\begin{array}{l}\text { Brazilian Archives of Biology } \\
\text { and Technology }\end{array}$ & $\begin{array}{l}\text { Assessment of the potential implementation of solar energy } \\
\text { generation in state schools in Curitiba/PR }\end{array}$ \\
\hline Santos et al. & 2019 & $\begin{array}{l}\text { Brazilian Archives of Biology } \\
\text { and Technology }\end{array}$ & $\begin{array}{l}\text { Analysis of solar photovoltaic energy potential in Brazilian } \\
\text { hydroelectric reservoirs through floating panels }\end{array}$ \\
\hline Sorgato et al. & 2018 & Renewable Energy & $\begin{array}{l}\text { Technical and economic evaluation of thin-film CdTe building- } \\
\text { integrated photovoltaics (BIPV) replacing façade and rooftop } \\
\text { materials in office buildings in a warm and sunny climate }\end{array}$ \\
\hline
\end{tabular}

Silva et al. (2019) em seu estudo onde analisa o processo de licenciamento ambiental para incrementação de usinas solares fotovoltaicas de grande porte no Brasil, em 27 regulamentos estaduais que estabelecem os requisitos de triagem que submetem o EIA (Estudo de impacto ambiental) a essas usinas, constataram que existem critérios de limite desiguais para determinar se a planta passará por licenciamento simplificado ou processo regular. Os autores ressaltam que o país necessita de um planejamento ambiental adequado para licenciar usinas fotovoltaicas para produção em larga escala, sendo que essa produção centralizada de energia fotoelétrica possivelmente estará sujeita a intervenções do Ministério Público em relação aos impactos em comunidades tradicionais. Para os autores, as preocupações surgem em relação a ações regulatórias, visto que no Brasil não há nenhuma resolução do CONAMA (Conselho Nacional do Meio Ambiente) e legislação específica que trata dos critérios para licenciar usinas fotovoltaicas de grande porte.

No Brasil a legislação voltada para setor fotovoltaico abrange somente sistemas de produção elétrica para mini ou migro geração de forma distribuída e não centralizada, como podemos citar as resoluções normativas n.o 482 (em 2012) e a resolução n.ㅇ 687 (em 2015), pela Agência Nacional de Energia Elétrica (ANEEL), porém, o governo federal tem realizados leilões específicos para implementação de usinas fotovoltaicas no país em larga escala. Souza et al. (2016) destaca que as políticas energéticas chinesas por priorizarem os recursos renováveis para produção de energia limpa, principalmente, a fotovoltaica, tornase uma grande referência para o Brasil sob a questão de discutir a experiência com essa tecnologia, a fim de destacar a importância dos investimentos em implementação de energias renováveis nas economias emergentes e o papel decisivo dos Estados nacionais no avanço desse setor energético como estratégia de negócio eficiente.

No estado do Rio de Janeiro foi realizando um estudo sobre a complementariedade energética utilizando pequenas centrais hidrelétricas em combinação com energias renováveis como parques eólicos e painéis fotovoltaicos, com intuito de reduzir a demanda de energia produzida por fontes convencionais e enfraquecer a utilização de fontes de energia não renováveis. Essa pesquisa conduzida por Rosa et al. (2017), contatou que a combinação de recursos hídricos, fotovoltaicos e eólicos no Estado do Rio de Janeiro 
pode reduzir sua variabilidade diária em até $61 \%$, se comparada a fontes isoladas. Jong et al. (2019) avaliando o impacto das mudanças climáticas, devido ao aquecimento global, sobre a energia solar e eólica, constatou que o potencial de produção de energia solar pode aumentar significativamente nas regiões Sudeste e Nordeste do Brasil.

No estudo realizado por Figueirêdo et al. (2019) para avaliar o impacto da tecnologia fotovoltaica em aldeia indígenas, os resultados apresentaram de forma positiva em relação a essa fonte de energia renovável, pois geralmente em locais de difícil acesso ou em locais de mata a carência por energia elétrica é ainda muito alta, visto que em tais ambientes no Brasil geralmente não possui eletricidade.

Schmidt et al. também analisando um cenário energético, através de métodos de simulação matemática, com o objetivo de reduzir a produção de gases de efeito estufa no Brasil, utilizou um modelo de otimização considerando usinas fotovoltaicas, eólicas, térmicas e hidrelétricas. Os resultados obtidos pelos autores indicam que, devido o constante aumento na demanda de energia desde 2013 , apenas $2 \%$ da energia deve ser fornecido por termelétricas, a combinação entre energia hidrelétrica e fotovoltaica apresentou um cenário ideal com percentual de 37\%, 9\% de energia eólica e 50\% de geração de energia hidrelétrica. Em um cenário apenas hidrotérmico o risco de déficit energético aumenta dez vezes e a produção de energia térmica quatro vezes mais.

No estudo de simulação sobre energia fotovoltaica para um sistema de bombeamento de água realizado por Carvalho et al. (2018) obteve resultados benéficos em relação ao uso dessa tecnologia. 0 mesmo resultado positivo ocorreu no estudo de Pereira et al. (2018), onde foi utilizado sistemas fotovoltaicos para diminuir os gastos com energia elétrica em escolas estaduais paranaense, onde obteve uma economia de $\mathrm{R} \$ 435$ mil anuais.

No estudo apresentado por Santos et al. (2019) onde analisou o potencial de geração de energia fotovoltaica em reservatórios hidrelétricos utilizando painéis flutuantes (ocupando 10\% dos reservatórios, podendo ter números maiores em maiores percentuais dessas áreas), sendo que tais painéis foram aplicados nas usinas Sobradinho, Tucuruí, Balbina e Itaipu durante o período de 2015 e 2016. Os resultados constatados nas três usinas poderiam apresentar um aumento considerável na produção de energia com a inserção dos painéis flutuantes fotovoltaicos, os quais possivelmente superaria os valores reais de energia gerada durante $o$ ano.

Na pesquisa realizada por Sorgato et al. (2018) de caráter técnico-econômico, onde foi integrado telureto de cádmio de película de filme fino (CdTe) em módulos solares fotovoltaicos e implementado em fachada e telhado de um edifício comercial, sendo que a análise foi aplicada para seis cidades brasileira: Florianópolis (SC), Curitiba (PR), Rio de Janeiro (RJ), Brasília (DF), Belém (PA) e São Paulo (SP). Nesse estudo os autores constaram que as cidades de Belém e Rio de Janeiro tiveram o maior consumo anual líquido de energia para ar-condicionado, sendo que estas cidades apresentam maiores temperaturas, 26,9 e 23,9 graus centrígrados, respectivamente. Considerando a produção do sistema fotovoltaico CdTe, São Paulo obteve a menor produção energética, visto que para as demais cinco cidades o sistema desenvolvido atende aos níveis de consumo de prédio de escritórios. 
Silva et al. (2019) visando reduzir os custos dos produtos comercializados por uma industria de ferroligas para que a empresa obtenha maior competitividade no mercado externo, os autores desenvolveram um estudo de uma simulação de uma usina fotovoltaica com a finalidade de reduzir os gastos com eletricidade. Esses autores concluiram que o comparativo do custo nivelado de energia motrou que a usina fotovoltaica proposta tem um preço inferior ao das fontes convencionais tando no mercado livre como no mercado regulado, sendo que os resultados obtidos apresentaram uma boa atratividade do ponto de vista financeiro para essa tecnologia renovável. Os autores afirmam ainda que custo em reais/kWh de energia nesse sistema foi quase 30\% menor que o mercado livre de energia e até $60 \%$ menor que o mercado regulado. No quadro seguinte são apresentados os demais artigos consultados para revisão.

Quadro 3: Artigos selecionados

\begin{tabular}{|c|c|c|c|}
\hline Autores & Ano & Periódico & Títulos \\
\hline Carvalho et al. & 2019 & Solar Energy & $\begin{array}{l}\text { Technical-economic analysis of the insertion of PV power into a wind- } \\
\text { solar hybrid system }\end{array}$ \\
\hline Tonin et al. & 2018 & $\begin{array}{l}\text { Brazilian Archives of } \\
\text { Biology and Technology }\end{array}$ & $\begin{array}{l}\text { Characterization of grid-connected photovoltaic systems in Curitiba, } \\
\text { Brazil }\end{array}$ \\
\hline Francisco et al. & 2019 & $\begin{array}{l}\text { Revista Brasileira de } \\
\text { Gestão Urbana }\end{array}$ & $\begin{array}{l}\text { Influência de parâmetros meteorológicos na geração de energia em } \\
\text { painéis fotovoltaicos: um caso de estudo do Smart Campus Facens, SP, } \\
\text { Brasil }\end{array}$ \\
\hline Hioki et al. & 2019 & $\begin{array}{l}\text { Brazilian Archives of } \\
\text { Biology and Technology }\end{array}$ & Performance analysis of small grid connected photovoltaic systems \\
\hline Lopes et al. & 2019 & $\begin{array}{l}\text { Journal of Material Cycles } \\
\text { and Waste Management }\end{array}$ & $\begin{array}{l}\text { Energy potential using landfill biogas and solar photovoltaic system: a } \\
\text { case study in Brazil }\end{array}$ \\
\hline Brunini et al. & 2019 & $\begin{array}{l}\text { Revista Brasileirade } \\
\text { Ciencias Agrarias }\end{array}$ & Economic analysis of photovoltaic energy in irrigating lettuce crops \\
\hline Mariano et al. & 2018 & $\begin{array}{l}\text { Brazilian Archives of } \\
\text { Biology and Technology }\end{array}$ & $\begin{array}{l}\text { Analysis of shifting and reduction of the demand peak with the inserting } \\
\text { of photovoltaic systems in the Neoville's Headquarters of Federal } \\
\text { University of Technology - Paraná Curitiba Campus }\end{array}$ \\
\hline Pillot et al. & 2018 & Renewable Energy & $\begin{array}{l}\text { Grid parity analysis of distributed PV generation using Monte Carlo } \\
\text { approach: the Brazilian case }\end{array}$ \\
\hline Carvalho et al. & 2017 & $\begin{array}{l}\text { RAM. Revista de } \\
\text { Administração Mackenzie }\end{array}$ & $\begin{array}{l}\text { Financial Alternatives to Enable Distributed Microgeneration Projects } \\
\text { with Photovoltaic Solar Power }\end{array}$ \\
\hline Takigawa et al. & 2019 & Ingeniare & $\begin{array}{l}\text { Analysis of the financial viability of a photovoltaic system to a consumer } \\
\text { unit in South Brazil }\end{array}$ \\
\hline Carvalho et al. & 2019 & Nova Economia & $\begin{array}{l}\text { Impactos econômicos da ampliação do uso de energia solar residencial } \\
\text { em Minas Gerais }\end{array}$ \\
\hline Garcia et al. & 2018 & $\begin{array}{l}\text { Brazilian Archives of } \\
\text { Biology and Technology }\end{array}$ & $\begin{array}{l}\text { Solar energy for residential use and its contribution to the energy matrix } \\
\text { of the State of Paraná. }\end{array}$ \\
\hline Stedile et al. & 2018 & $\begin{array}{l}\text { Brazilian Archives of } \\
\text { Biology and Technology }\end{array}$ & $\begin{array}{l}\text { Comparison of the Performance of the Grid- Connected Photovoltaic } \\
\text { System Installed In the Brazilian Cities: Blumenau - SC and Curitiba- PR }\end{array}$ \\
\hline Mariano et al. & 2018 & $\begin{array}{l}\text { Brazilian Archives of } \\
\text { Biology and Technology }\end{array}$ & $\begin{array}{l}\text { The Photovoltaic Generation and its Energy Contribution and Demand } \\
\text { Shifting at the Center Headquarters of the Federal University of } \\
\text { Technology - Paraná - Campus Curitiba }\end{array}$ \\
\hline
\end{tabular}

No Brasil, a aplicação dos sistemas de geração de eletricidade apartir da tecnologia fotovoltaica estão cada vez mais sendo combinados a outras fontes de energia limpa como forma de complementação energética, os chamados sistemas híbridos, sendo uma alternativa viável considerando a questão econômica-financeira-ambiental. Todavia, Carvalho et al. (2019) ressalta que "para mitigar a falta de produção de energia, diversos tipos de fontes de energia foram combinados em um chamado sistema híbrido de energia; no entanto, algumas análises econômicas devem ser realizadas para avaliar se o negócio híbrido é financeiramente viável".

Stedile et al. (2018) também realizando estudo sobre oito sistemas fotovoltaicos conectado à rede, 
sendo três deste na cidade de Curitiba (PR) e cinco em Blumenau (SC), constatou que os $3 \mathrm{kWp}$ instalados em uma residência em Curitiba produziu aproximadamente 704,13 kWh/kWp (em sete meses durante o ano de 2017), tal desempenho teve como justificativa as ótimas condições de instalação - desvio azimutal muito próximo de zero e inclinação próxima à latitude da cidade (25 para Curitiba), já o sistema instalado em Blumenau apresentou baixo desempenho devido as configurações do sistema e menor irradiância solar. O Brasil possui ótimas condições para implementação de sistemas fotovoltaicos devido aos elevados índices de irradiância em todo país, Francisco et al. (2019) ao analisar os parâmetros meteorológicos para implantação de painéis fotovoltaicos na cidade de São Paulo (SP), contatou-se que a umidade e a temperatura são fatores negativos para geração de energia fotovoltaica, no entanto, a velocidade do vento tendo sido bastante significativa para o aumento da geração de energia. Assim como, no estudo realizado por Hioki et al. (2019), no qual obteve resultados similares ao estudo de Francisco et al. (2019.

Lopes et al. (2019) avaliou um sistema híbrido de geração de energia (fotovoltaica e biogás) em um aterro sanitário localizado em Itajubá (MG), no qual pode gerar uma quantidade consideravél de energia durante o período de recebimento de resíduos sólidos urbanos e após o encerramento do aterro, onde o sistema biogás produziria um total de 162,1 GWh por 30 anos e o sistema solar 15,1 GWh por 25 anos de operação, sendo que o sistema apresentou um desempenho satisfatório de 83,1\% (sistema solar de minigeração). Apesar do alto fator de sustentabilidade ambiental envolvido nesse sistema de produção de energia, fica explícito os altos custos operacionais na compra e instalação dos sistemas fotovoltaicos, sendo um dos principais entraves na implementação dessa tecnologia no país.

Brunini et al. (2019) analisando a implementação da tecnologia fotovoltaica para irrigação de uma lavoura de alface utilizando um sistema teste por gotejamento de água, onde esses autores realizarão uma avaliação econômico-financeira dessa tecnologia em comparação com um sistema convencional de eletricidade. Durante a avaliação os autores constataram que o sistema de energia fotovoltaica reduziu o custo com eletricidade em $83,80 \%$, quando comparado ao sistema convencional. Os autores verificaram ainda que a energia consumida pelo sistema fotovoltaico durante os períodos de teste foi $76,4 \mathrm{~W}$, tendo um consumo elétrico de 2,8 kWh com tempo de ciclo de irrigação de 37 horas (ciclo da alface). Em contra partida, o sistema convencional teve um consumo de energia de 99,0 W em um período de 23,43 horas com uma taxa total de consumo de 2,6 kWh de energia para o ciclo da alface, no entanto, o sistema fotovoltaico teve um gasto de 2,13 vezes maior que o sistema convencional de energia com, respectivamente, $\mathrm{R} \$ 783,30$ e $\mathrm{R} \$ 367,00$.

Em seu estudo, Carvalho et al. (2019), avaliaram um sistema eólico-fotovoltaico, através de uma simulaçao hipotética de uma usina híbrida instalada no Nordeste brasileiro como modelo teste utilizando oito aerogeradores eólicos com potência nominal de 2,7 MW instaladas em duas localidades diferentes e 19200 painéis solares com 250W de potência nominal, onde constataram que "à medida que os arranjos fotovoltaicos são inseridos no complexo, as chances de um negócio bem sucedido diminuem, embora não se torne inviável se for utilizada uma quantidade baixa de potência nominal fotovoltaica". Conforme os autores, no sistema híbrido composto por $60 \%$ de energia fotovoltaica ocasiona certa diminuição na 
probabilidade de VPL (Valor Presente Líquido) favorável, sendo que os sistemas solares em larga escala são ainda bastante caros no Brasil e a produção de energia fotoelétrica é proporcionalmente baixa em comparação a geração eólica em unidades.

Mariano et al. (2018) realizando um estudo para expansão de um sistema fotovoltaico em uma universidade federal em Curitiba (PR) com o objetivo de analisar o deslocamento e picos de demanda, onde foi projetado um sistema com capacidade de $2008,38 \mathrm{kWp}$ (resultando em um aumento de $436,88 \%$ do sistema existente), com o desenvolvimento do sistema verificou-se que haveria injeção do excedente de energia fotogerada mesmo nos dias de baixa disponibilidade de irradiação solar e poderia ser estendido para outras partes da universidade. Realizando uma nova aplicação de sistemas fotovoltaicos na mesma instituição de ensino, Mariano et al. (2018), expandiram um sistema fotovoltaico de 2,1 kWp de potência interligado à rede desde 2011 nessa instituição para 645,81 kWp, onde obtiveram um aumento de potência nominal de 307,5 vezes comparado ao anterior. O objetivo do estudo foi analisar o perfil da curva de demanda e o consumo dos prédios sede. Nota-se que os dois estudos foram muito semelhantes, através da aplicação da mesma metodologia, no entanto, obtiveram resultados positivos que comprovam o potencial da tecnologia fotovoltaica como recurso energético promissor da sustentabilidade ambiental.

Pillot et al. (2018) analisaram a qualidade da rede de geração distribuída no Brasil conectado ao sistema convencional de energia elétrica, usando uma abordagem probabilística de Monte Carlos, onde constaram que sistemas utilizando potência nominal de $4 \mathrm{~kW}$ podem ser viáveis, mas apenas por questão de dependência de financiamento de capital. Considerando a questão de financiamento de dívidas, segundos os autores, apenas sistemas com capacidade nominal acima de $7 \mathrm{~kW}$ apresentam níveis de confiança significativos. No Brasil é de grande importância a expansão da matriz elétrica do país, devido sobretudo aos impactos ambientais ocasionados pelos sistemas convencionais de produção, no entanto, para que tais objetivos sejam alcançados é necessário maior flexibilidade do modelo regulatório brasileiro, “com mudanças que possibilitem a expansão da oferta de energia renovável, com resultados econômicos positivos para a acessibilidade tarifária" (CARVALHO et al., 2017).

Takigawa et al. (2019) analisando um projeto de um sistema geração de energia fotovoltaica para identificar os diversos aspectos que influencia o retorno financeiro, através da simulação de vários cenários, percebeu-se que a variação da tarifa no decorrer dos anos tem forte influência no retorno financeiro, pois estar diretamente relacionado com os custos de aquisição dos equipamentos que compõe o sistema. Os autores evidenciaram também que o modo de aquisição através de financiamento e a possibilidade de venda de excedente de energia são fatores ainda não regulamentados no Brasil, porém, podem contribuir efetivamente para o tempo de aquisição e retorno financeiro do sistema.

Segundo a pesquisa realizada por Carvalho et al. (2017), onde analisaram os impactos econômicos da adoção de painéis fotovoltaicos por um conjunto de 10 famílias mineiras, constataram que apenas as famílias dos grupos de renda a partir de 5 salários-mínimos per capita aderem a essa fonte alternativa de energia. Os autores ressaltam que famílias com renda per capita entre 5 e 10 salários-mínimos são mais beneficiadas pela aquisição dessa fonte de energia, em termos de ganhos de consumo. Os autores explicam 
ainda, que o consumo de eletricidade das famílias que compõem esse grupo familiar é integralmente fornecido pelo sistema fotovoltaico, sem mais gastos provenientes da concessionária de energia, visto que a economia de energia corresponde a um percentual maior da renda dessas famílias e, é por tal razão, que o efeito positivo da adoção dos sistemas fotovoltaicos para essa classe é mais proeminente. Seria de grande importância à existência de programas de financiamento para os interessados em adquirir seus próprios centros de microgeração (GARCIA et al., 2018).

No entanto, ainda conforme Carvalho et al. (2019), o principal efeito da adoção de energia fotovoltaica em residências familiares está relacionado ao aumento do consumo propiciado pela renda extra gerada, que, por sua vez, afeta setores produtivos, pois considerando o período de 2017 a 2036 pode constatar que nos primeiros anos iniciais ocorreria uma redução na arrecadação de imposto estadual (Circulação de Mercadorias e Serviços - ICMS), sendo que nesse período as famílias teriam gastos maiores com o financiamento do que com a redução da conta de energia elétrica e somente depois de alguns anos começaria a ocorrer ganho líquido de renda e o consequentemente aumento da atividade econômica, o que resultaria na arrecadação tributária para o governo. Os autores notaram também que a arrecadação de impostos sobre as vendas começaria a aumentar durante o ano de 2030, ou seja, após 13 anos depois. 0 quadro a seguir segue com os resultados dos artigos consultados.

Quadro 4: Artigos selecionados

\begin{tabular}{|c|c|c|c|}
\hline Autores & Ano & Periódico & Títulos \\
\hline Silveira et al. & 2018 & $\begin{array}{l}\text { Brazilian Archives of } \\
\text { Biology and Technology }\end{array}$ & Feasibility Study through Grid-Connected Photovoltaic Systems in Curitiba \\
\hline Scolari et al. & 2018 & $\begin{array}{l}\text { Brazilian Archives of } \\
\text { Biology and Technology }\end{array}$ & $\begin{array}{l}\text { Mapping and Characterization of the Grid-connected Photovoltaic } \\
\text { Systems in the City of Curitiba: Preliminary Results }\end{array}$ \\
\hline Ribeiro et al. & 2018 & $\begin{array}{l}\text { Brazilian Archives of } \\
\text { Biology and Technology }\end{array}$ & $\begin{array}{l}\text { Generation of Photovoltaic Solar Energy. Evaluation of the Demand Curve } \\
\text { with the Insert of Grid-connected Photovoltaic Power System at } \\
\text { CINDACTA II, Curitiba-PR, Brazil. }\end{array}$ \\
\hline Silveira et al. & 2019 & $\begin{array}{l}\text { Brazilian Archives of } \\
\text { Biology and Technology }\end{array}$ & $\begin{array}{c}\text { Study of the Potential of Photovoltaic Microgeneration and } \\
\text { Minigeneration from the Grid-Connected Photovoltaic Systems Installed } \\
\text { at UTFPR Curitiba Campus }\end{array}$ \\
\hline Viana et al. & 2018 & Applied Energy & $\begin{array}{l}\text { Analysis of demand response and photovoltaic distributed generation as } \\
\text { resources for power utility planning }\end{array}$ \\
\hline Tiago Filho et al. & 2016 & $\begin{array}{c}\text { Solar Energy Materials } \\
\text { and Solar Cells }\end{array}$ & $\begin{array}{l}\text { Study of the energy balance and environmental liabilities associated with } \\
\text { the manufacture of crystalline Si photovoltaic modules and deployment in } \\
\text { different regions }\end{array}$ \\
\hline $\begin{array}{l}\text { Urbanetz Junior et } \\
\text { al. }\end{array}$ & 2018 & $\begin{array}{l}\text { Brazilian Archives of } \\
\text { Biology and Technology }\end{array}$ & $\begin{array}{c}\text { Analysis of the Operation of Photovoltaic Systems Installed at Federal } \\
\text { University of Technology - Paraná in Curitiba }\end{array}$ \\
\hline
\end{tabular}

Para Silveira et al. (2018) os sistemas fotovoltaicos conectados à rede de energia elétrica é uma solução eficaz como fonte de geração distribuída, no qual pode contribuir para o aumento da disponibilidade de energia elétrica para o Brasil e apesar do investimento ser de alto custo, a economia em longo prazo na conta de energia do consumidor o torna um sistema vantajoso. Scolari et al. (2018) realizou um mapeamento da utilização dos sistemas fotovoltaicos na cidade de Curitiba (PR), observou que é nas residências familiares onde se encontra $78 \%$ do total de paneis fotovoltaicos da cidade com $52 \%$ da potência total instalada. Os autores ressaltam que os sistemas residenciais possuem uma potência média de 2,97 kW, já os sistemas comerciais e industriais têm uma potência média de 9,48 kW e 9,69 kW respectivamente. Tonin et al. (2018) ao realizar uma caracterização dos sistemas fotovoltaicos conectado à 
rede em Curitiba (PR), verificou que até 2017 a capital paranaense possuía 204 sistemas fotovoltaicos instalados com potência total de 997,4 kW em operação (o estado possuía 1031 sistemas instalados e operando com 6,65 MWp), sendo que 82\% (2,98 MWp) estavam instalados em residências familiares. Os resultados obtidos por Urbanetz Junior et al. (2018) e Ribeiro et al. (2018) apresentaram resultados similares aos autores citados neste parágrafo. Viana et al. (2018) abordaram em seu estudo que a demanda ainda não explorada e a produção de energia nos sistemas de distribuição baseados em preços (DR) e geração distribuída de energia fotovoltaica (PVDG), são recursos estratégicos para o planejamento de concessionárias de energia, no qual pode possibilitar o deferimento de investimento na expansão da rede e redução do consumo de energia na subestação.

Os consumidores residenciais podem reduzir a conta de energia com DR, dependendo de sua flexibilidade para alterar o comportamento de consumo ou por meio da implantação do PVDG (VIANA et al., 2018), o que contribuiria para redução dos gases do efeito estufa (GEE) através da estimulação do incremento de produção de eletricidade de fontes limpas, porém, isso requer uma avaliação da regulamentação brasileira para estimular a políticas de preços com base no uso racional de energia elétrica, ou seja, controle de demanda em picos de consumo ou mesmo em que os usuários recebem uma remuneração pela participação em programas de redução de demanda. Outro fator relacionado à tecnologia solar fotovoltaica é sob a questão da produção de dióxido de carbono $\left(\mathrm{CO}_{2}\right)$ um dos gases mais poluentes do ar atmosférico, no entanto, essa questão mantém relação somente com as formas de produção dos painéis fotovoltaicos e seus componentes periféricos necessários para a instalação desses sistemas sendo responsáveis pelo seu funcionamento. Tiago Filho et al. (2016) estudou os passivos ambientais associados à fabricação de módulos fotovoltaicos de Si cristalino, os custos energéticos e as emissões de $\mathrm{CO}_{2}$ geradas. O principal resultado obtido na pesquisa desse autor foi que quanto maior o fator de emissão $\left(\mathrm{CO}_{2}\right)$ do país produtor do painel, maior o tempo necessário para a redução das emissões devido à sua produção, sendo que "o sistema analisado se limitou ao processo de produção da célula fotovoltaica, à placa de vidro e ao caixilho de alumínio desde a extração da matéria-prima até a montagem final do painel fotovoltaico, com exceção das informações relativas ao transporte do material".

A conversão de minério de alumínio, bauxita (isto é, alumina impura, Al2O3) ao metal de alumínio é o processo industrial que consome mais energia do que qualquer outro na produção de painéis fotovoltaicos (TIAGO FILHO et al., 2016). Os autores destaca também os impactos gerados na produção e descarte final de baterias de chumbo-ácido (principalmente no descarte) utilizados no armazenamento de energia de células fotovoltaicas (utilizado sistema isolado de produçao de energia), sendo que essas baterias têm uma vida útil inferior aos paineis solares entre 2 a 5 anos e geralmente durante a vida útil de um painel, cerca de quatro baterias são utilizadas e descartadas no meio ambiente.

Ainda conforme Tiago Filho et al. (2016), o tempo necessário de operaçao de painel solar para amortizar os custos relativos de produção de eletricidade é 2,31 anos para sistemas interligados a rede convencional e 3,23 anos para sistemas isolados de produção de energia, neste cenário, após os paineis atingir o equilíbrio energético, os mesmos tem 22,69 (com baterias) e 21,76 (sem baterias) anos de vida útil 
ambiental. No caso brasileiro, caso os paineis fotovoltaicos sejam produzidos no país, somente os sistemas fotovoltaicos isolados apresentaram alto teor de geração de $\mathrm{CO}_{2}$ com taxa de $0,821 \mathrm{kgCO} / \mathrm{kWh}$, já os sistemas interligados as redes convencionais apresentaram baixo fator de poluição com 0,121 kgCO2/kWh. Portanto, os autores concluem que o fator de emissão (EF) de carbono é que definará o total de anos que os paineis fotovoltaicos efetivamente operarão na produção de energia totalmente limpa como, por exemplo, a produção painéis solares no Brasil considerando o sistema interligado brasileiro $(\mathrm{EF}=0,121$ $\mathrm{kgCO} 2 / \mathrm{kWh}$ ) e usado em um país com fator de emissão de $0,3[\mathrm{kgCO} e \mathrm{eq} / \mathrm{kWh}]$, ele operará produzindo energia efetivamente limpa por 22 anos, sendo que o inverso também é verdadeiro, ocorrendo somente o aumento ou a diminuição de anos (considerando os 25 anos de vida útil do painel solar).

\section{CONCLUSÃO}

A contribuição da energia solar fotovoltaica para a matriz elétrica no Brasil ainda se encontra com potencial muito baixo em comparação com outras fontes de energia, com taxa inferior a $2 \%$ do total gerado no país. No entanto, desde 2015 o país vem apresentando aumento na taxa de geração e potencial instalado, devido, sobretudo ações governamentais para implementação dessa tecnologia em todo o território nacional, como podemos citar as publicações das resoluções normativas n.o 482 (em 2012) e n.으 687 (em 2015), além dos leilões específicos realizados pelo governo federal para implementação de usinas fotovoltaicas em larga escala. Podemos constatar através deste estudo de revisão, que em relação a sistemas energia fotovoltaicos para produção de eletricidade em larga escala ainda carece de regulações apropriadas para concessão de licenças ambientais, porém, o licenciamento dessa fonte de energia apresenta critérios de limite desiguais para determinar se a planta passará por licenciamento simplificado ou processo regular.

A geração de energia fotovoltaica com base na literatura pesquisa é considerada uma fonte alternativa de energia limpa sem ocasionar impactos ambientais apenas quando os sistemas se encontram em efetiva operação. Sendo que, somente em relação à produção dos painéis solares e dos seus componentes periféricos necessários para montagem produzem impactos ambientais, o que o torna grande emissor de dióxido de carbono $\left(\mathrm{CO}_{2}\right)$ em potencial na atmosfera. Esses impactos surgem desde a extração das matérias-primas (alumínio, silício, vidro etc.) até fabricação dos componentes para montagem das placas solares, visto que os sistemas fotovoltaicos que funcionam interligados a baterias de chumboácido são mais poluentes, devido ao descarte final dessas baterias. Podemos constatar também através deste estudo, que a maioria das pesquisas encontradas utilizam sistemas fotovoltaicos em modelos de teste de simulação matemática combinadas a outras fontes de energia renováveis, os chamados sistemas híbridos de produção de energia, tendo maior predominância nos sistemas eólicos-fotovoltaicos. Sendo que no Brasil, os sistemas fotovoltaicos conectados à rede de energia elétrica estão mais disseminados do que os sistemas fotovoltaicos isolados, principalmente, na utilização em residências familiares em centros urbanos.

A tecnologia fotovoltaica no Brasil já estar bastante disseminada em uma enorme variedade de 
aplicações e certamente serão umas das fontes de energia limpa mais utilizadas no futuro. No entanto, o principal empecilho atualmente para que a energia produzida à base de irradiação solar tenha uma maior disseminação em todo o território nacional é sobre a questão dos custos de financiamento dos paneis solares que são muitos caros, o que o torna ainda inacessível para toda a população do país, devido à questão salarial das famílias brasileiras serem bastantes baixas. Pois a população do Brasil é composta em grande parte por pessoas de classe mais pobres.

\section{REFERÊNCIAS}

ABPA. Associação Brasileira de Proteína Animal. Relatório Anual de 2020: carne de frango e ovos. São Paulo, 2020.

AQUINO, P. S. A.; SILVA, J. D. C.. Geração solar. Brazilian Applied Science Review, v.3, n.1, p.370-378, 2019.

AZEVEDO, D.. Revisão de Literatura, Referencial Teórico, Fundamentação Teórica e Framework Conceitual em Pesquisa: diferenças e propósitos. 2020.

BEN. Balanço Energético Nacional 2016: ano base 2015, 2016.

BEN. Balanço Energético Nacional 2020: ano base 2019, 2020.

BRUNINI, R. G.; SILVA, A. B.; PAULA, V. R.; OLIVEIRA, J. C.. Economic analysis of photovoltaic energy in irrigating lettuce crops. Revista Brasileirade Ciencias Agrarias, v.14, n.4, 2019. DOI: http://doi.org/10.5039/agraria.v14i4a6539

CARSTENS, D. D. S.; CUNHA, S. K.. Challenges and opportunities for the growth of solar photovoltaic energy in Brazil. Energy Policy, v.125, p.396-404, 2019. DOI: https://doi.org/10.1016/j.enpol.2018.10.063

CARVALHO, D. B.; GUARDIA, E. C.; LIMA, J. W. M.. Technicaleconomic analysis of the insertion of PV power into a windsolar hybrid system. Solar Energy, v.191, p.530-539, 2019. DOI: https://doi.org/10.1016/j.solener.2019.06.070

CARVALHO, F. I. A.; ABREU, M. C. S.; CORREIA NETO, J. F. Financial Alternatives To Enable Distributed Microgeneration Projects With Photovoltaic Solar Power. Revista de Administração Mackenzie, v.18, n. 1, p.120-147, 2017. DOI:http://dx.doi.org/10.1590/167869712017/administraca o.v18n1p120-147

CARVALHO, M. M.; MAGALHÃES, A. S.; DOMINGUES, E. P.. Impactos econômicos da ampliação do uso de energia solar residencial em Minas Gerais. Nova Economia, v.29, n.2, p. 459-485, 2019. DOI: https://doi.org/10.1590/0103$\underline{6351 / 4719}$

CARVALHO, R. D.; DALSASSO, R. L.; GUEDES, T. L.; SANTOS, J. A. C.. Sizing optimization in solar photovoltaic water pumping system: Model validation in a pilot unit located in the rural community of Rio Belo, Orleans/SC, Brazil. Engenharia Sanitaria e Ambiental, v.23, n.6, p.1153-1162, 2018. DOI:http://dx.doi.org/10.1590/s141341522018160134

CETEM. Silício grau solar: uma revisão das tecnologias de produção. 26 ed. Rio de Janeiro: CETEM/MCTIC, 2019.
DIDONÉ, E. L.; WAGNER, A.; PEREIRA, F. O. R.. Estratégias para edifícios de escritórios energia zero no Brasil com ênfase em BIPV TT - Strategies towards Net Zero Energy Office Buildings in Brazil with emphasis on BIPV. Ambiente Construído, v.14, n.3, p.27-42, 2014. DOI: http://dx.doi.org/10.1590/S1678-86212014000300003

FIGUEIRÊDO NETO, G. S.; ROSSI, L. A.. Photovoltaic energy in the enhancement of indigenous education in the Brazilian Amazon. Energy Policy, v.132, p.216-222, 2019. DOI: https://doi.org/10.1016/j.enpol.2019.05.037

FRANCISCO, A. C. C.; VIEIRA, H. E. M.; ROMANO, R. R.; ROVEDA, S. R. M. M.. Influência de parâmetros meteorológicos na geração de energia em painéis fotovoltaicos: um caso de estudo do Smart Campus Facens, SP, Brasil. Revista Brasileira de Gestão Urbana, v.11, p.1-15, 2019. DOI:

http://dx.doi.org/10.1590/21753369.011.e20190027

GARCIA, G.; NOGUEIRA, E. F.; BETINI, R. C. Solar energy for residential use and its contribution to the energy matrix of the State of Paraná. Brazilian Archives of Biology and Technology, v. 61, 2018. DOI: http://doi.org/10.1590/16784324-smart-2018000510

GIL, A. C.. Como elaborar projetos de pesquisa. 6 ed. São Paulo: Atlas, 2017.

HIOKI, A. T.; SILVA, V. R. G. R.; VILELA JUNIOR, J. A.; LOURES, E. F. R.. Performance analysis of small grid connected photovoltaic systems. Brazilian Archives of Biology and Technology, v.62, p.1-10, 2019. DOI: http://doi.org/10.1590/1678-4324-smart-2019190018

JONG, P.; BARRETO, T. B.; TANAJURA, C. A. S.; KOULOUKOUI, D.; OLIVEIRA-ESQUERRE, K. P.; KIPERSTOK, A.; TORRES, E. A.. Estimating the impact of climate change on wind and solar energy in Brazil using a South American regional climate model. Renewable Energy, v.141, p.390-401, 2019. DOI: https://doi.org/10.1016/j.renene.2019.03.086

LOPES, M. M.; COBAS, V. R. M.; BARROS, R. M.; LORA, E. E. S.; SANTOS, I. F. S.. Energy potential using landfill biogas and solar photovoltaic system: a case study in Brazil. Journal of Material Cycles and Waste Management, v.21, n.6, p.15871601, 2019. DOI: https://doi.org/10.1007/s10163-01900904-7

MACHADO, C. T.; MIRANDA, F. S.. Photovoltaic solar energy: A briefly review. Revista Virtual de Quimica, v.7, n.1, p.126143, 2015. DOI: https://doi.org/10.5935/1984$\underline{6835.20150008}$ 
MARIANO, J. D.; URBANETZ JUNIOR, J.. Analysis of shifting and reduction of the demand peak with the inserting of photovoltaic systems in the Neoville's Headquarters of Federal University of Technology - Paraná Curitiba Campus. Brazilian Archives of Biology and Technology, v.61, 2018.

MARIANO, J. D.; URBANETZ JUNIOR, J.. The Photovoltaic Generation and its Energy Contribution and Demand Shifting at the Center Headquarters of the Federal University of Technology - Paraná - Campus Curitiba. Brazilian Archives of Biology and Technology, v.61, p.1-11, 2018. DOI: https://doi.org/10.1590/1678-4324-smart-2018000280

NOBRE, P.; PEREIRA, E. B.; LACERDA, F. F.; BURSZTYN, M.; HADDAD, E. A.; LEY, D.. Solar smart grid as a path to economic inclusion and adaptation to climate change in the Brazilian Semiarid Northeast. International Journal of Climate Change Strategies and Management, v.11, n.4, p. 499-517, 2019. DOI: https://doi.org/10.1108/IJCCSM-092018-0067

PEREIRA, J. S.; RIBEIRO, D. A.; LUCENA, F. H.; URBANETZ JUNIOR, J.. Assessment of the potential implementation of solar energy generation in state schools in Curitiba/PR. Brazilian Archives of Biology and Technology, v.61, 2018. DOI: https://doi.org/10.1590/1678-4324-smart-2018000300

PILLOT, B.; SIQUEIRA, S.; DIAS, J. B.. Grid parity analysis of distributed PV generation using Monte Carlo approach: the Brazilian case. Renewable Energy, v.127, p.974-988, 2018.

PIRES, P. V. L.; LIMA, T. C.; OLIVEIRA, G. V.. Estudo da conexão de módulos solares fotovoltaicos à rede elétrica de distribuição. ANALECTA: Centro de Ensino Superior de Juiz de Fora, v.5, n.5, p.1-18, 2019. DOI: https://doi.org/10.1016/j.renene.2018.05.032

ROSA, C. O. C. S.; COSTA, K. A.; CHRISTO, E. S.; BERTAHONE, P. B.. Complementarity of hydro, photovoltaic, and wind power in Rio de Janeiro State. Sustainability, v.9, n.7, p.1-12, 2017. DOI: https://doi.org/10.3390/su9071130

SANTOS, F. R.; MARIANO, J. A.; SESTREM JUNIOR, J. A.; URBANETZ JUNIOR, J.. Analysis of solar photovoltaic energy potential in Brazilian hydroelectric reservoirs through floating panels. Brazilian Archives of Biology and Technology, v.62, 2019. DOI: https://doi.org/10.1590/16784324-smart-2019190012

SAUER, I. L.; QUEIROZ, M. S.; MIRAGAYA, J. C. G.; MASCARENHAS, R. C.; QUINTINO JÚNIOR, A. R.. Energias renováveis: ações e perspectivas na Petrobras. Bahia Análise \& Dados, v.16, n.1, p.9-22, 2006.

SCHMIDT, J.; CANCELLA, R.; PEREIRA JR., A. O.. An optimal mix of solar PV, wind and hydro power for a low-carbon electricity supply in Brazil. Renewable Energy, v.85, n.2016, p.137-147, 2016. DOI:

http://dx.doi.org/10.1016/j.renene.2015.06.010

FRANCISCO, A. C. C.; VIEIRA, H. E. M.; ROMANO, R. R.; M. M.; ROVEDA, S. R.. Mapping and Characterization of the Gridconnected Photovoltaic Systems in the City of Curitiba: Preliminary Results. Brazilian Archives of Biology and Technology, v.61, 2018. DOI: https://doi.org/10.1590/21753369.011.e20190027

SILVA, F. R.; SILVA, O. R.; LIMA, F. L.; BASTOS, W. F.. Difusão da inovação tecnológica : um estudo sobre a difusão da energia elétrica fotovoltaica no Brasil. Revista Tecnologia, v. 40, n.2, p.1-12, 2019. DOI:

https://doi.org/10.5020/23180730.2019.9810

SILVA, G. D. P.; MAGRINI, A.; TOLMASQUIM, M. T.; BRANCO, D. A. C.. Environmental licensing and energy policy regulating utility-scale solar photovoltaic installations in Brazil: status and future perspectives. Impact Assessment and Project Appraisal, v.37, n.6, p.503-515, 2019. DOI: https://doi.org/10.1080/14615517.2019.1595933

SILVA, L. G. L.; DIAS, W. P.; VIEIRA, C. B.; ASSIS, P. S.. Solar photovoltaic energy applied to ferroalloy industry. International Engineering Journal - REM, v.72, n.2, p.251255, 2019. DOI: https://doi.org/10.1590/037044672018720021

SILVEIRA, C. O.; MOREIRA, A. R.; MOREIRA, B. L. P.; URBANETZ JUNIOR, J.. Feasibility Study through GridConnected Photovoltaic Systems in Curitiba. Brazilian Archives of Biology and Technology, v.61, 2018. DOI: https://doi.org/10.1590/1678-4324-smart-2018000230

SORGATO, M. J.; SCHNEIDER, K.; RÜTHER, R.. Technical and economic evaluation of thin-film CdTe building-integrated photovoltaics (BIPV) replacing façade and rooftop materials in office buildings in a warm and sunny climate. Renewable Energy, v.118, p.84-98, 2018. DOI:

https://doi.org/10.1016/i.renene.2017.10.091

SOUZA, A. B.; OLIVEIRA, A. L.. Benefícios ambientais da energia fotovoltaica. Revista Interface Tecnológica, v.16, $\mathrm{n}$. 2, p.287-298, 2019. DOI:

https://doi.org/10.31510/infa.v16i2.644

SOUZA, L. E. V.; CAVALCANTE, A. M. G.. Towards a sociology of energy and globalization: Interconnectedness, capital, and knowledge in the Brazilian solar photovoltaic industry.

Energy Research and Social Science, v.21, p.145-154, 2016. DOI: http://dx.doi.org/10.1016/j.erss.2016.07.004

STEDILE, R.; TONIN, F. S.; URBANETZ JUNIOR, J.. Comparison of the Performance of the Grid- Connected Photovoltaic System Installed In the Brazilian Cities: Blumenau - SC and Curitiba- PR. Brazilian Archives of Biology and Technology, v.61, 2018. DOI: https://doi.org/10.1590/1678-4324-smart$\underline{2018000028}$

TAKIGAWA, F. Y. K.; ARANHA NETO, E. A. C.; FERNANDES, R. C.; CAMPOS, D.; CARDOSO, M.. Analysis of the financial viability of a photovoltaic system to a consumer unit in South Brazil. Ingeniare, v.27, n.1, p.131-141, 2019. DOI: http://dx.doi.org/10.4067/S0718-33052019000100131

TIAGO FILHO, G. L.; ROSA, C. A.; BARROS, R. M.; SANTOS, I. F. S.; SILVA, F. G. B.. Study of the energy balance and environmental liabilities associated with the manufacture of crystalline Si photovoltaic modules and deployment in different regions. Solar Energy Materials and Solar Cells, v. 144, p. 383-394, 2016. DOI: http://dx.doi.org/10.1016/j.solmat.2015.09.023

TONIN, F. S.; URBANETZ JUNIOR, J.. Characterization of gridconnected photovoltaic systems in Curitiba, Brazil. Brazilian Archives of Biology and Technology, v.61, 2018. DOI: https://doi.org/10.1590/1678-4324-smart-2018000250

TORRES, D. G. B.. Células fotovoltaicas: desenvolvimento e 
as três. Revista Técnico-Científica do CREA-PR, v.1, p.1-6, 2019.

TORRES, R. C.. Energia solar fotovoltaica como fonte alternativa de geração de energia elétrica em edificações residenciais. Dissertação (Mestrado em Engenharia Mecânica) - Universidade de São Paulo, São Carlos, 2012.

URBANETZ, I. V.; MOURA NETTO, A.; SCOLARI, B.; LEITE, V.; URBANETZ, J.. Current panorama and 2025 scenario of photovoltaic solar energy in Brazil. Brazilian Archives of Biology and Technology, v.62, p.1-7, 2019. DOI: https://doi.org/10.1590/1678-4324-smart-2019190011
VIANA, M. S.; MANASSERO JUNIOR, G.; UDAETA, M. E. M. Analysis of demand response and photovoltaic distributed generation as resources for power utility planning. Applied Energy, v.217, p.456-466, 2018. DOI: https://doi.org/10.1016/j.apenergy.2018.02.153

VILLALVA, M. G.; GAZOLI, J. R.. Energia solar fotovoltaica: conceitos e aplicações. São Paulo: Editora Érica, 2012.

A CBPC - Companhia Brasileira de Produção Científica (CNPJ: 11.221.422/0001-03) detém os direitos materiais desta publicação. Os direitos referem-se à publicação do trabalho em qualquer parte do mundo, incluindo os direitos às renovações, expansões e disseminações da contribuição, bem como outros direitos subsidiários. Todos os trabalhos publicados eletronicamente poderão posteriormente ser publicados em coletâneas impressas sob coordenação da Sustenere Publishing, da Companhia Brasileira de Produção Científica e seus parceiros autorizados. Os (as) autores (as) preservam os direitos autorais, mas não têm permissão para a publicação da contribuição em outro meio, impresso ou digital, em português ou em tradução. 